\title{
Clinical and Economic Burden of Antineutrophil Cytoplasmic Antibody-associated Vasculitis in the United States
}

\author{
Karina Raimundo, Amanda M. Farr, Gilwan Kim, and George Duna
}

ABSTRACT. Objective. To describe the prevalence of major relapse and healthcare costs among patients with granu-
lomatosis with polyangiitis (GPA); to find patients with microscopic polyangiitis (MPA) in adminis-
trative databases, because no MPA diagnosis code exists; and to describe the clinical and economic
burden associated with MPA.
Methods. Adults $\geq 18$ yrs) with $\geq 2$ diagnoses of GPA [International Classification of
Diseases-9-Clinical Modification (ICD-9-CM 446.4)] during 2009-2013 were extracted from the
Truven Health MarketScan Commercial and Medicare Supplemental databases. Evidence of major
relapse (based on the Birmingham Vasculitis Activity Score) and healthcare costs were collected during
12-month and 24-month followup periods. Adults with $\geq 2$ diagnoses of unspecified arteritis
(ICD-9-CM 447.6) were found as potential patients with MPA and additional criteria based on clinical
input were applied to refine the sample. Major relapse-associated conditions and healthcare costs in
the 6 months pre- and post-diagnosis were measured. Costs were inflated to 2013 US\$. Results. A total of 2784 patients with GPA were found and $18.7 \%$ experienced a major relapse in the 12-month followup period. The patients with a major relapse incurred higher average all-cause (12-month: $\$ 88,065$ vs $\$ 30,682 ; \mathrm{p}<0.0001)$ and GPA-related costs $(12$-month: $\$ 61,636$ vs $\$ 15,748$; $\mathrm{p}<0.0001)$ than patients without a relapse. Trends were consistent over the 24-month followup period. There were 612 incident patients with MPA. Following MPA diagnosis, healthcare costs nearly doubled $(\$ 30,166$ vs $\$ 56,642 ; \mathrm{p}<0.0001)$.

Conclusion. In a real-world setting, patients with GPA who experience major relapse have higher economic burden, compared to patients without a relapse. MPA diagnosis was associated with nearly a 2-fold increase in healthcare costs. (First Release November 1 2015; J Rheumatol 2015;42:2383-91; doi:10.3899/ jrheum.150479)

\section{Key Indexing Terms: \\ ANTINEUTROPHIL CYTOPLASMIC ANTIBODY-ASSOCIATED VASCULITIS GRANULOMATOSIS WITH POLYANGIITIS HEALTHCARE EXPENDITURES MICROSCOPIC POLYANGIITIS RECURRENCE}

Granulomatosis with polyangiitis (GPA) and microscopic polyangiitis (MPA) are the 2 most common antineutrophil cytoplasmic antibody (ANCA)-associated vasculitides $(\mathrm{AAV})^{1,2}$. AAV are characterized by necrotizing inflammation of small vessels, although medium vessels may also be involved in some cases ${ }^{3}$. GPA involves granulomatous inflammation of the respiratory tract and necrotizing vasculitis of the small vessels of the kidneys ${ }^{3}$. There is similar

From Genentech Inc., South San Francisco, California; Truven Health Analytics, Bethesda, Maryland, USA.

Funded by Genentech Inc. K. Raimundo and G. Duna are employees of Genentech. A.M. Farr and G. Kim are employees of Truven Health Analytics, which received funding to conduct this analysis from Genentech Inc.

K. Raimundo, MS, Health Economist, US Medical Affairs, Genentech Inc.; A.M. Farr, MPH, Research Leader, Truven Health Analytics; G. Kim, PharmD, MS, Sr. Research Analyst, Truven Health Analytics; G. Duna, MD, Rheumatologist with Immunology Medical Affairs, US Medical Affairs, Genentech Inc.

Address correspondence to K. Raimundo, 1 DNA Way, South San Francisco, California 94080,USA.E-mail: Raimundo.karina@gene.com Full Release Article. For details see Reprints/Permissions at jrheum.org Accepted for publication August 6, 2015. organ involvement in MPA, though the inflammation is nongranulomatous ${ }^{3}$. Although these diseases are rare, they are associated with increased mortality compared to the general population ${ }^{4}$.

Although the exact prevalence of the diseases is unknown, studies conducted in Europe, the United States, and Australia have reported GPA prevalence estimates ranging from 24 to 160 cases per million and MPA prevalence estimates ranging from 9 to 39.1 cases per million people ${ }^{5}$. In the United States, an observational study conducted in Montana and published in 2005 reported a GPA prevalence of 90 per million people and an annual incidence rate of 8.6 per million. The study found a prevalence of MPA of 30 per million and an annual incidence of 2.9 per million 6 .

AAV disease severity can be measured using a modified version of the Birmingham Vasculitis Activity Score $(\mathrm{BVAS})^{7}$. The BVAS is an itemized list of signs and symptoms associated with vasculitis, classified as minor (defined as important manifestations of active disease) or major (defined as an immediate threat to a vital organ or to

Personal non-commercial use only. The Journal of Rheumatology Copyright $\odot$ 2016. All rights reserved. 
life $)^{7}$. Disease status is classified into categories ranging from severe disease/flare, also known as relapse, to remission, where no active disease is present ${ }^{7,8}$. A severe disease/flare is indicated by the new presentation or worsening of a major BVAS item in the month prior to evaluation; whereas remission is indicated by no newly present, worsening, or persistent BVAS items ${ }^{7}$.

Treatment for GPA and MPA consists of induction therapy to achieve remission and maintenance therapy to sustain disease remission ${ }^{8,9}$. For many years, a variety of cytotoxic agents [cyclophosphamide (CYC), methotrexate (MTX), or azathioprine (AZA)] and corticosteroids have remained the treatments of choice for the majority of patients with GPA or MPA $^{9}$. While treatment with these agents imparts symptomatic benefit and reduces the risk of relapses ${ }^{9}$, none of these therapies is effective for all patients and each carries its own risk of side effects ${ }^{10}$. In 2011, rituximab (RTX), a chimeric anti-CD20 monoclonal antibody, in combination with corticosteroids, became the first therapy approved by the US Food and Drug Administration to induce disease remission for adults with GPA or MPA ${ }^{11,12}$.

However, high relapse rates, indicative of disease progression, remain a major clinical concern among patients with GPA and MPA. Several analyses using older data have evaluated the prevalence of relapse among patients with these conditions in non-experimental settings, although the definition of relapse differed within each analysis ${ }^{13,14,15,16}$. Over median followup times ranging from 42 to 47 months, about $35 \%$ to $60 \%$ of patients experienced a relapse ${ }^{13,14,15,16}$.

To date, current real-world data on the relapse rates and healthcare resource use and costs associated with GPA among patients in the United States are unavailable. We hypothesize that GPA patients with a relapse may require higher healthcare use, resulting in higher healthcare costs compared to those without a relapse. Therefore, the purpose of this retrospective cohort analysis was to estimate the prevalence of relapse, healthcare use, and costs associated with GPA. Exploratory objectives of our study were to develop an algorithm to find MPA patients in administrative claims databases, because no diagnosis code specific to MPA exists, and to quantify the clinical and economic burden associated with MPA.

\section{MATERIALS AND METHODS}

Data source. This was a longitudinal, retrospective, observational cohort study using claims data extracted from Truven Health MarketScan Commercial and Medicare Supplemental Databases for the period 2009 to 2013. Both the Commercial and Medicare databases contain integrated patient-level pharmacy and medical (inpatient and outpatient) claims of several million individuals annually, covered under a variety of fee-for-service, fully capitated, and partially capitated health schemes. Claims contain International Classification of Diseases-9-Clinical Modification (ICD-9-CM) diagnosis and procedure codes, Current Procedural Terminology codes, Healthcare Common Procedure Coding System codes, and National Drug Codes. Results from laboratory tests are only available for a subset of patients and were not used in this analysis. In addition to claims, the databases contain enrollment files that include demographic information and insurance plan information. All study data were compliant with the Health Insurance Portability and Accountability Act of 1996. Because our study used only de-identified patient data, it was exempt from Institutional Review Board approval.

Patient selection criteria. For the patients with GPA, adults ( $\geq 18$ yrs) were found with at least 2 medical claims with a diagnosis of GPA (ICD-9-CM diagnosis code 446.4) in any diagnosis position from July 1, 2010, through June 30, 2012. Patients were categorized as incident or prevalent GPA cases To be included in the analysis as an incident patient, the following 3 criteria must have been met: continuous enrollment for at least 12 months prior to their first GPA diagnosis (index date); (2) no claims with a GPA diagnosis or a diagnosis of unspecified arteritis (ICD-9-CM 447.6) in the 12 months prior to index date; and (3) 12 months of continuous enrollment following the index date (12-month followup period). Continuous enrollment is required to ensure that all medical and pharmacy services that generate a health insurance claim during those time periods will be identified. A subsample of incident patients had 24 months of continuous enrollment after the index date (24-month followup period). Patients who did not meet the pre-index date diagnosis (i.e., they did not have a 12-month clean period with a GPA diagnosis) or continuous enrollment criteria (i.e., they did not have 12 months of continuous enrollment prior to first GPA diagnosis) for incident cases were included in the prevalent patient sample. Because their diagnosis may not be clinically meaningful (as first diagnosis is for the incident patients), the index date for prevalent patients was defined as the date of most recent diagnosis of GPA with 12 months of followup so that the most recent data were used. A subsample of prevalent patients with 12 months of followup prior to index date was evaluated for outcomes over a 24-month followup period ( \pm 12 mos around the index date). The 12 months before and after were analyzed because the "index date" for prevalent patients represents an encounter with the healthcare system but does not represent initial diagnosis as it does for incident patients.

For the patients with MPA, those with at least 2 claims with a diagnosis of unspecified arteritis (ICD-9-CM diagnosis code 447.6) between July 1, 2010, and June 30, 2012 were found. Unspecified arteritis diagnosis was used because no diagnosis code specific to MPA exists. Patients were required to be $\geq 18$ years old at first diagnosis of unspecified arteritis (index date), have continuous enrollment for 6 months prior to the index date (pre-period) with no claims for unspecified arteritis (so as to gather incident cases), and have 6 months of continuous enrollment following the index date (post-period). After finding these potential patients with MPA, prevalence of diagnoses of conditions associated with major relapse based on the BVAS ${ }^{7}$ was measured. Based on these results and clinical input, patients with a claim with a diagnosis for renal failure, glomerulonephritis, or hemoptysis during the pre- or post-period were included, because these diagnoses are characteristic of severe MPA disease. Alveolar hemorrhage could not be gathered from claims data because no ICD-9-CM diagnosis code for the condition exists. Patients with a GPA diagnosis or hepatitis B or hepatitis C diagnosis during the pre- or post-period were excluded to avoid the misclassification of patients with vasculitis associated with hepatitis $\mathrm{B}$ or $\mathrm{C}$ as patients with MPA.

Study variables. Major relapse events were defined by a combination of diagnoses of GPA and other conditions based on the $\mathrm{BVAS}^{7}$ and medication use. The proportion of patients experiencing a relapse and the total number of relapses per patient during 12-month and 24-month followup periods were evaluated. For incident patients, claims were evaluated for major relapse starting after the index date plus 30 days so as to avoid recording initial diagnosis of GPA as a major relapse event. A major relapse event was defined as 1 of the following events: (1) a nondiagnostic inpatient or emergency room (ER) claim with a GPA diagnosis in any position; or (2) at least 2 nondiagnostic outpatient office visit claims with a GPA diagnosis in a 30-day period; or (3) a nondiagnostic inpatient, ER, or outpatient claim with a diagnosis of a relapse-associated condition based on the major items in the BVAS [acute/chronic renal failure, gangrene, scleritis, retinal exudates/hemorrhage, sensorineural deafness, mesenteric ischemia, hemoptysis (no

Personal non-commercial use only. The Journal of Rheumatology Copyright (C) 2016. All rights reserved 
ICD-9-CM diagnosis for alveolar hemorrhage exists), respiratory failure, meningitis, cord lesion, stroke, cranial nerve palsy, sensory peripheral neuropathy, motor mononeuritis multiplex $]^{7}$ in any position. The event must have then been followed by a claim for oral/intravenous (IV) CYC, RTX, or corticosteroids (oral with prednisone-equivalent dose $>20 \mathrm{mg}$ or IV) within 30 days. In addition, there must have been no claims for these drugs and no evidence of relapse in the 30 days prior to the aforementioned event.

All-cause and GPA-related healthcare use and costs were measured during followup. GPA-related claims were defined as those with a diagnosis of GPA or a major relapse-associated condition as defined above in any position or a claim for a GPA medication (oral and IV corticosteroids, oral and IV CYC, MTX, AZA, leflunomide, RTX, mycophenolate mofetil, tumor necrosis factor blocker biologics, and trimethoprim/sulfamethoxazole) based on the Healthcare Common Procedure Coding System and National Drug Codes. Specific use measures included the presence and number of inpatient admissions, ER visits, outpatient use (outpatient office visits, and other outpatient care), and pharmacy prescriptions. Other outpatient care included outpatient services such as radiological and biochemical laboratory tests. The number of GPA-related outpatient office visits to primary care physicians and specialists was measured during followup as was the presence of claims for specific GPA medications.

Healthcare costs (all-cause and GPA-related) were reported overall and by the types of aforementioned services. Healthcare costs were based on paid amounts of adjudicated claims, including insurer and health plan payments, as well as patient cost-sharing. All-cause and GPA-related healthcare costs are reported as 2013 US\$, adjusted using the Medical Care component of the Consumer Price Index ${ }^{17}$

Patient demographic variables including age, sex, and geographical location (U.S. census division) were measured on index date. Clinical characteristics measured in the 12-month pre-period for the incident GPA and during the post-period for all patients with GPA included the Deyo adaptation of the Charlson Comorbidity Index ${ }^{18}$ and severe manifestations of disease based on the presence of a claim with a diagnosis of major relapse-associated conditions, based in turn on the major items in the BVAS ${ }^{7}$. Whereas major relapse events were not evaluated in the 30 days after index date for incident patients to avoid classifying initial diagnosis as a relapse, the presence of diagnoses of major relapse-associated conditions were measured starting on the index date to describe manifestation of disease. Note that diagnoses were only part of the algorithm for identifying relapse. Supplemental Figure 1A-B depicts the study period for the GPA analysis (available online at jrheum.org).

For the MPA analysis, demographic characteristics were measured at index date. In addition, presence of diagnosis of a major relapse-associated condition to describe disease manifestations and all-cause healthcare use and costs were measured in the 6 months before and after diagnosis. Relapse events were not evaluated. Costs were also measured over 3-month intervals to examine trends.

Statistical analysis. Costs were compared between GPA patients with and without a major relapse using Wilcoxon and Mann-Whitney tests. The difference in costs for patients with MPA pre- and post-diagnosis was compared using a Wilcoxon signed-rank sum test, and use was compared using McNemar's tests. P values $<0.05$ were considered statistically significant. Incremental cost differences and $95 \%$ CI were calculated. No other statistical comparisons were made.

\section{RESULTS}

GPA cohort. Applying patient selection criteria yielded a study population of 2784 patients, 569 in the incident cohort and 2215 in the prevalent cohort. Of these, 309 (54.3\%)
A

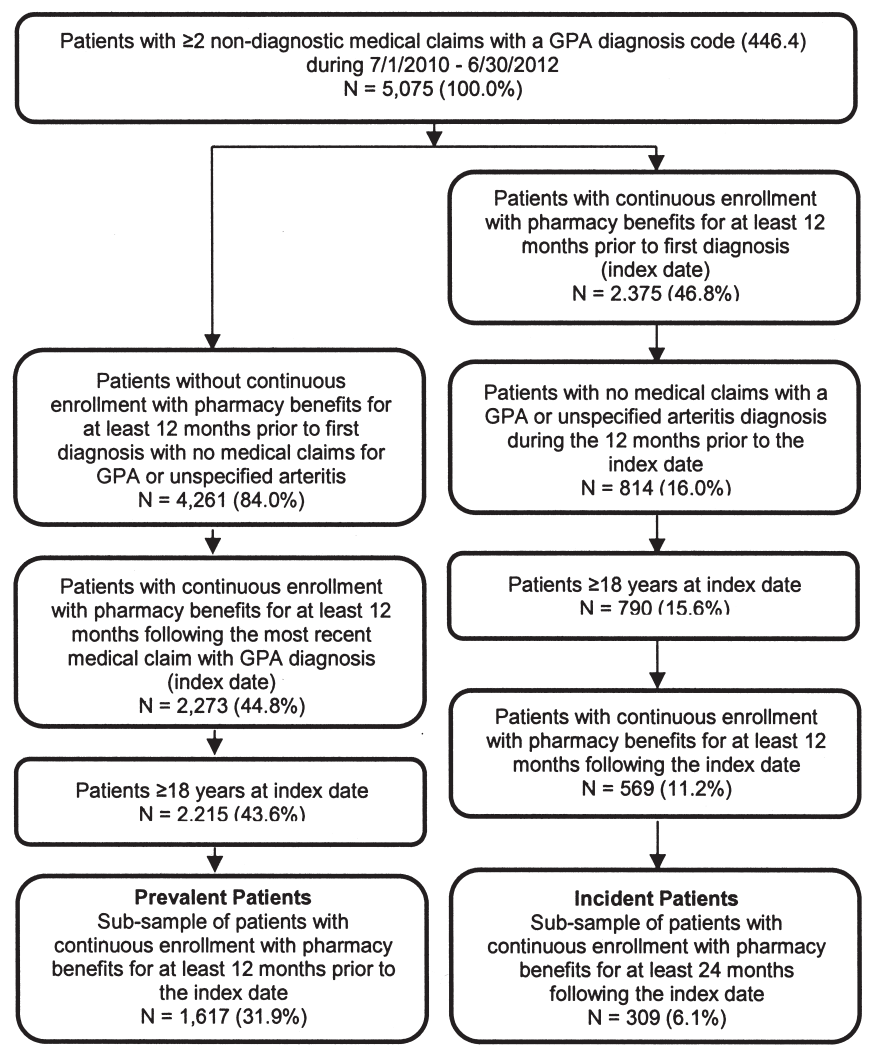

B

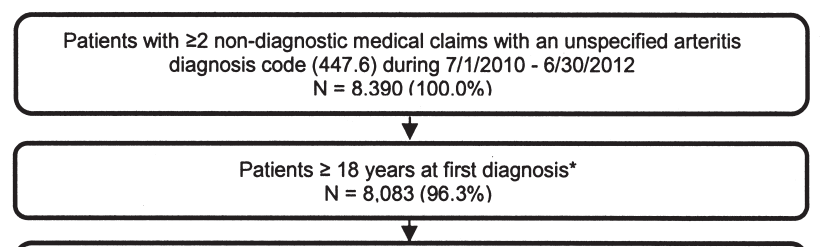

Patients with continuous enrollment with pharmacy benefits for at least 6 months prior to first diagnosis $N=6.694(79.8 \%)$

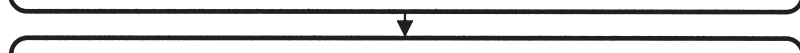

Patients with continuous enrollment with pharmacy benefits for at least 6 months following the first diagnosis $\mathrm{N}=5.923(70.6 \%)$

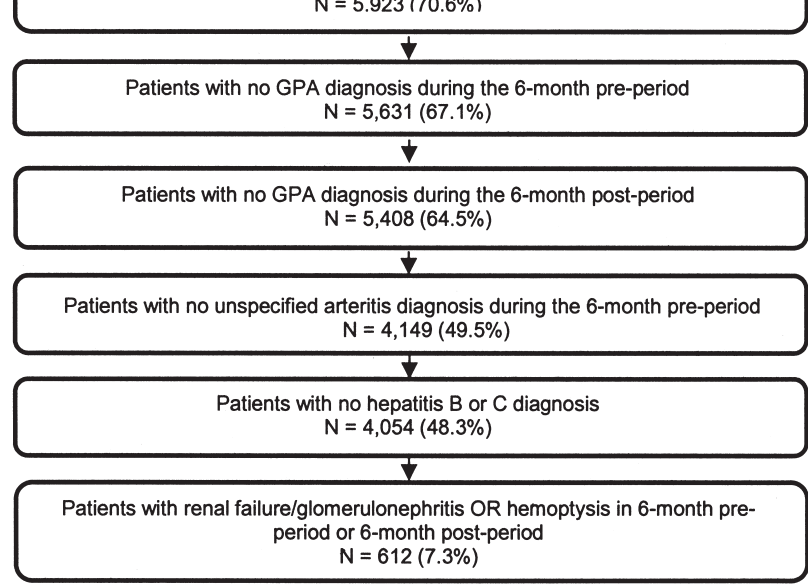

Figure 1. A. Attrition for prevalent and incident GPA cohorts. B. Attrition for the incident MPA cohort. GPA: granulomatosis with polyangiitis; MPA: microscopic polyangiitis.

Personal non-commercial use only. The Journal of Rheumatology Copyright $\subset$ (2016. All rights reserved. 
incident cases and $1617(73.0 \%)$ prevalent cases had continuous enrollment with pharmacy benefits for at least 24 months (Figure 1A).

Overall, $79.0 \%$ of patients with GPA were above 45 years of age. More than half of the patients were females $(54.0 \%)$. Incident and prevalent cases differed in several characteristics. Compared to the prevalent GPA cohort, those in the incident GPA cohort were older (mean age: 57.9 vs $56.0 \mathrm{yrs}$ ), and a lower proportion of them were female $(51.1 \%$ vs $55.1 \%$; Table 1).

Within the year prior to GPA diagnosis, $23.2 \%$ of incident cases were diagnosed with acute or chronic renal failure. Of those patients, less than half $(37.1 \%)$ had their first renal failure diagnosis within 6 months of GPA diagnosis. Over the 12-month pre-period, $7 \%$ were diagnosed with either hemoptysis or respiratory failure (lung-related conditions). The majority of these patients (91.3\% diagnosed with hemoptysis and $77.3 \%$ diagnosed with respiratory failure) had their first relapse-associated diagnosis within 6 months before their first GPA diagnosis. The proportion of patients with these conditions increased in the followup periods. Acute/chronic renal failure, lung-related conditions, and sensorineural deafness were the most common major relapse-related diagnoses during the 12- and 24-month followup periods among incident and prevalent GPA cohorts (12-month followup: Table 1; 24-month followup: Supplemental Table 1, available online at jrheum.org).

During the 12-month followup period, $18.7 \%$ of patients

Table 1. Demographic and clinical characteristics of patients with GPA during the 12-month pre-period and 12-month followup.

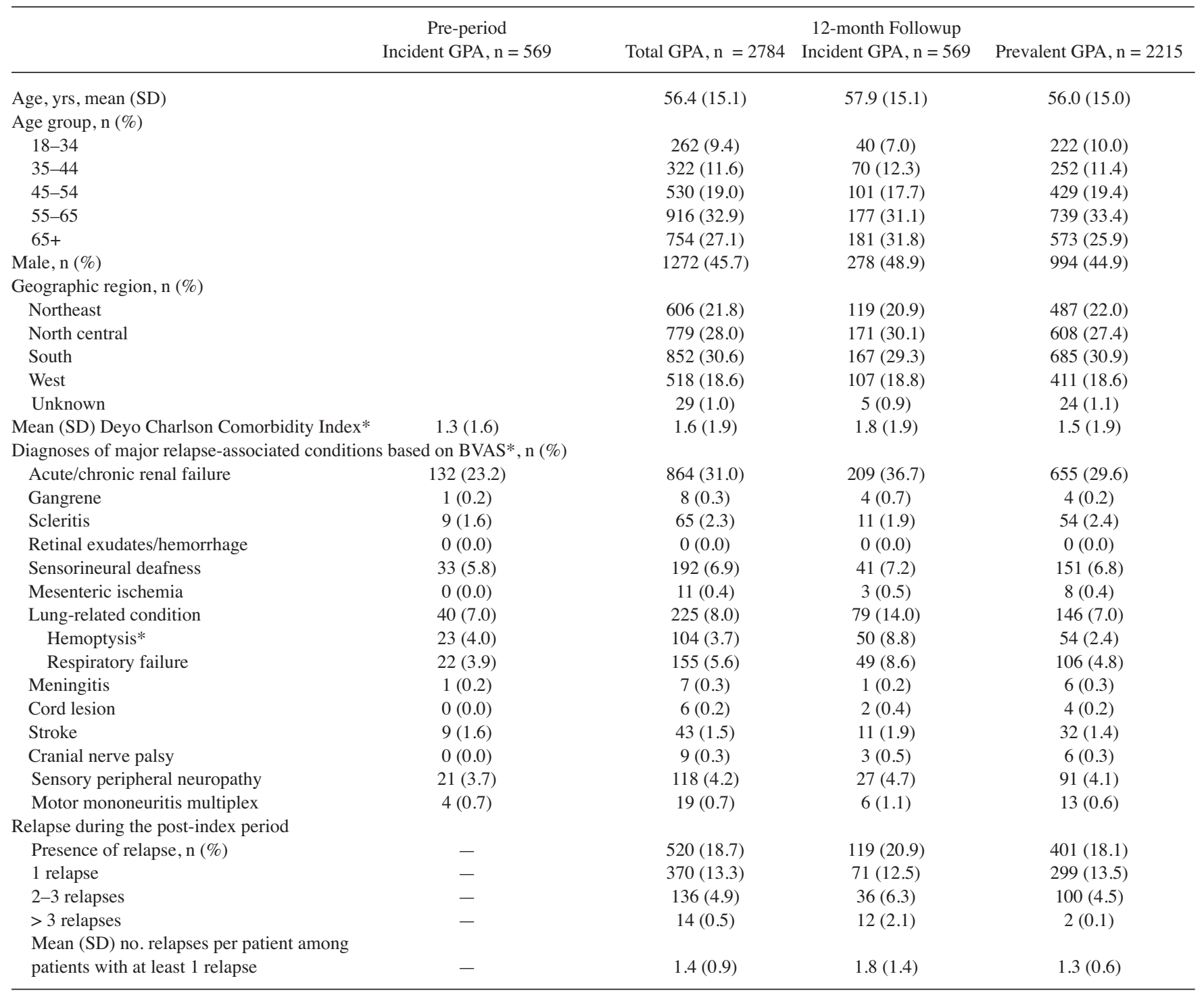

* Patients with hemoptysis (ICD-9-CM 786.30,786.39) were identified because no diagnosis codes were available for alveolar hemorrhage. BVAS: Birmingham Vasculitis Activity Score; GPA: granulomatosis with polyangiitis; MPA: microscopic polyangiitis; ICD-9-CM: International Classification of Diseases-9- Clinical Modification. 
experienced $\geq 1$ major relapse. Of those patients, $28.8 \%$ experienced $\geq 2$ major relapses in that period. The prevalence of relapse was slightly higher among incident cases (20.9\%) compared to prevalent patients (18.1\%). A similar pattern was noted among the prevalent GPA cohort (Table 1; and Supplemental Table 1, available online at jrheum.org).

During the 12-month followup, the proportion of patients with an all-cause inpatient admission was greater among incident patients $(42.2 \%)$ compared to prevalent patients (21.0\%). The same trend was found for all-cause ER visits over the 12 months after index (incident: $40.2 \%$ vs prevalent: 29.9\%). Regarding GPA-related use, 38.8\% of incident patients and $17.5 \%$ of prevalent patients had a GPA-related inpatient admission. GPA-related ER visits were uncommon, with only $7 \%$ of patients experiencing at least 1 during the 12 -month followup period. About $90 \%$ of patients had at least 1 GPA-related office visit during the 12-month followup and $86.6 \%$ had at least 1 GPA-related other outpatient service, including dialysis. The average number of GPA-related outpatient office visits to a primary care physician was 1.3 per year [SD 2.6; incident: 1.5 (SD 3.1); prevalent: 1.2 (SD 2.5)] and to a rheumatologist was 1.4 [SD 2.6; incident: 1.3 (SD 2.9); prevalent: 1.5 (SD 2.6)]. The average numbers of visits were smaller to a nephrologist [all: 0.2 visits (SD 0.9); incident: 0.3 (SD 1.3); prevalent: 0.2 (SD 0.8)] and to a pulmonologist [all: 0.3 visits (SD 1.0); incident: 0.4 (SD 1.4); prevalent: 0.3 (SD 0.9)].

Also during the 12-month followup period, 2364 (84.9\%) patients with GPA had at least 1 claim for a GPA-related treatment (469 incident patients and 1895 prevalent patients). Oral corticosteroids were the most commonly used therapy, with $69.2 \%$ of patients with at least 1 claim for the medication class during the followup. Of treated patients,
19.3\% had claim(s) for oral or IV corticosteroids only. Overall, 7\% of patients had a claim for oral CYC, but this proportion was higher among incident patients than prevalent patients (17.2\% vs $4.1 \%)$. There were 599 patients (21.5\%) who had at least 1 claim for AZA and 266 (9.6\%) who had at least 1 claim for RTX.

The mean total all-cause and GPA-related costs were $\$ 41,400$ (SD: $\$ 87,537$ ) and \$24,319 (SD: \$74,362) during the 12-month followup. On average, 59\% of all-cause costs per patient with GPA were associated with GPA. Other outpatient services (including dialysis) and inpatient hospitalization were the largest cost drivers (Figure 2, and Supplemental Table 2, available at jrheum.org). When stratified by relapse status, GPA patients with a relapse incurred higher total all-cause and GPA-related costs than those without a relapse (all-cause: $\$ 88,065$ vs $\$ 30,682$, $p<0.0001$; GPA-related: $\$ 61,636$ vs $\$ 15,748, \mathrm{p}<0.0001$; Figure 3, and Supplemental Table 3 available online at jrheum.org).

MPA analysis. The final study cohort comprised 612 patients (Figure 1B). The mean (SD) average age at diagnosis was 61.5 years (16.1) and $62.1 \%$ were female. The most common pre/post-period major relapse-associated condition was acute/chronic renal failure followed by lung-related conditions. About $60 \%$ of patients had a claim with a diagnosis of a major relapse-associated condition in the 6 months prior to first unspecified arteritis diagnosis. All-cause healthcare resource use increased after MPA diagnosis. Similarly, the all-cause healthcare costs almost doubled following an MPA diagnosis $[\$ 30,166$ vs $\$ 56,642$, incremental cost difference $\$ 26,476$ (95\% CI: $\$ 17,678-\$ 35,274), p<0.0001]$ with costs related to inpatient admissions being the main driver before and after diagnosis $[\$ 15,344$ vs $\$ 34,776$, incremental cost difference $\$ 19,432$ (95\% CI: \$11,586-\$27,277), p < 0.0001;

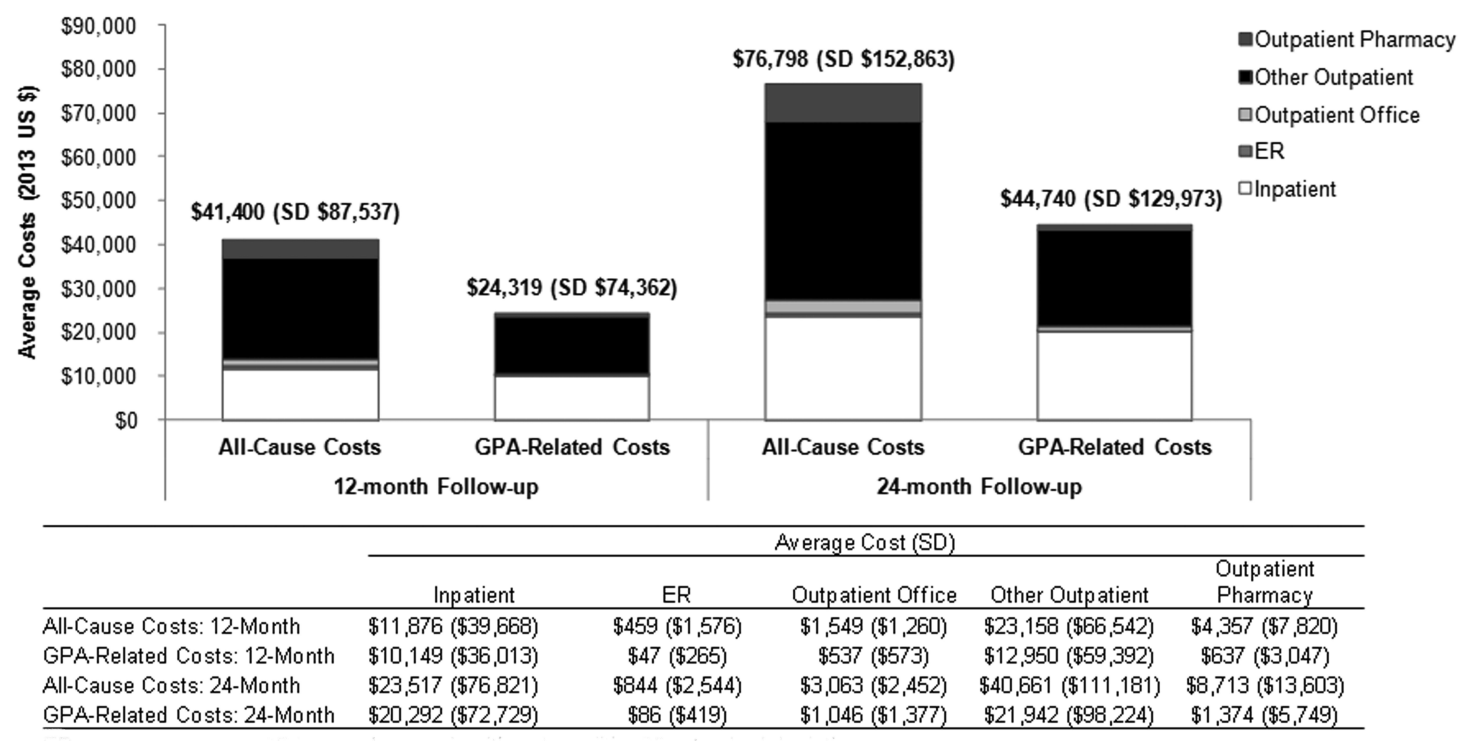

Figure 2. All-cause and GPA-related healthcare costs of patients with GPA in the 12-month and 24-month followup periods. GPA: granulomatosis with polyangiitis; ER: emergency room.

Personal non-commercial use only. The Journal of Rheumatology Copyright $\subset$ 2016. All rights reserved. 
Table 2. Clinical characteristics of patients with MPA during the pre- and post-period. All costs are US\$.

\begin{tabular}{|c|c|c|c|}
\hline Characteristics & $\begin{array}{c}6 \text { Months } \\
\text { Pre-diagnosis, } \\
n=612\end{array}$ & $\begin{array}{c}6 \text { Months } \\
\text { Post-diagnosis, } \\
n=612\end{array}$ & $\mathrm{p}^{*}$ \\
\hline \multicolumn{4}{|c|}{ Diagnoses of major relapse-associated conditions based on BVAS*, n (\%) } \\
\hline Acute/chronic renal failure & $329(53.8)$ & $435(71.1)$ & \\
\hline Gangrene & $10(1.6)$ & $23(3.8)$ & \\
\hline Scleritis & $2(0.3)$ & $3(0.5)$ & \\
\hline Retinal exudates/hemorrhage & $0(0.0)$ & $2(0.3)$ & \\
\hline Sensorineural deafness & $14(2.3)$ & $18(2.9)$ & \\
\hline Mesenteric ischemia & $7(1.1)$ & $10(1.6)$ & \\
\hline Lung-related condition & $61(10.0)$ & $99(16.2)$ & \\
\hline Hemoptysis** & $24(3.9)$ & $44(7.2)$ & \\
\hline Respiratory failure & $43(7.0)$ & $68(11.1)$ & \\
\hline Meningitis & $3(0.5)$ & $2(0.3)$ & \\
\hline Cord lesion & $0(0.0)$ & $1(0.2)$ & \\
\hline Stroke & $30(4.9)$ & $49(8.0)$ & \\
\hline Cranial nerve palsy & $1(0.2)$ & $1(0.2)$ & \\
\hline Sensory peripheral neuropathy & $30(4.9)$ & $39(6.4)$ & \\
\hline Motor mononeuritis multiplex & $0(0.0)$ & $3(0.5)$ & \\
\hline Any relapse-associated diagnosis & $370(60.5)$ & $476(77.8)$ & \\
\hline \multicolumn{4}{|c|}{ All-cause healthcare use and costs } \\
\hline \multicolumn{4}{|l|}{ Inpatient admissions } \\
\hline \multicolumn{4}{|l|}{$\begin{array}{l}\text { At least } 1 \text { inpatient admission, } \\
\mathrm{n}(\%)\end{array}$} \\
\hline Mean (SD) inpatient costs & $\begin{array}{c}\$ 15,344 \\
(\$ 43,332)\end{array}$ & $\begin{array}{c}\$ 34,776 \\
(\$ 95,590)\end{array}$ & $<0.0001$ \\
\hline \multicolumn{4}{|l|}{ ER visits } \\
\hline At least 1 ER visit, n (\%) & $256(41.8)$ & $290(47.4)$ & 0.0295 \\
\hline Mean (SD) ER costs & $\$ 893(\$ 2610)$ & $\$ 1019(\$ 2483)$ & 0.0144 \\
\hline \multicolumn{4}{|l|}{ Outpatient office visits } \\
\hline \multicolumn{3}{|l|}{ At least 1 outpatient office } & 0.1138 \\
\hline \multicolumn{4}{|l|}{ Mean (SD) outpatient office } \\
\hline \multicolumn{4}{|l|}{ Other outpatient services } \\
\hline $\begin{array}{l}\text { At least } 1 \text { other outpatient } \\
\text { service, } \mathrm{n}(\%)\end{array}$ & $601(98.2)$ & $612(100.0)$ & N/A \\
\hline $\begin{array}{l}\text { Mean (SD) other outpatient } \\
\text { service costs }\end{array}$ & $\begin{array}{l}\$ 10,498 \\
(\$ 25,452)\end{array}$ & $\begin{array}{l}\$ 16,725 \\
(\$ 32,036)\end{array}$ & $<0.0001$ \\
\hline \multicolumn{4}{|l|}{ Outpatient pharmacy } \\
\hline \multicolumn{3}{|l|}{ At least 1 prescription drug } & 0.3938 \\
\hline \multicolumn{4}{|l|}{ Mean (SD) prescription drug } \\
\hline \multicolumn{4}{|l|}{ Total } \\
\hline Mean (SD) total costs & $\begin{array}{l}\$ 30,166 \\
(\$ 58,253)\end{array}$ & $\begin{array}{c}\$ 56,642 \\
(\$ 108,227)\end{array}$ & $<0.0001$ \\
\hline
\end{tabular}

* Statistical comparisons were done only for use and costs measures. ** Patients with hemoptysis (ICD-9-CM 786.30, 786.39) were identified because no diagnosis codes were available for alveolar hemorrhage. BVAS: Birmingham Vasculitis Activity Score; ER: emergency room; MPA: microscopic polyangiitis; ICD-9-CM: International Classification of Diseases-9-Clinical Modification.

Table 2]. Average costs increased prior to diagnosis, peaked in the 3 months following diagnosis, and then decreased (Figure 4, and Supplemental Table 4 available online at jrheum.org).

\section{DISCUSSION}

Our current study demonstrated that in 1 year of followup, one-fifth of patients with GPA experienced a major relapse event. In addition, the average healthcare costs incurred over the followup periods for patients with GPA who had a major relapse were substantially higher than for those without any relapse. Patients with MPA were found in the administrative databases using a new algorithm, developed through clinical input, because of a lack of a specific ICD-9-CM code for MPA. Receiving a diagnosis of MPA was associated with an increase in all-cause healthcare resource use and costs, driven primarily by an increase in costs of inpatient admission and experienced in the first 3 months after diagnosis.

Several other observational analyses have noted high relapse rates among patients with ANCA-associated vasculitides $^{13,14,15,16}$. Koldingsnes and Nossent observed that $60 \%$ of patients experienced a relapse, with a median time to relapse of 18 months $^{13}$. In a United States-based observational study, Hogan, et al reported that relapse occurred in $42 \%$ of patients who had achieved remission, over a median of 44 months of followup ${ }^{14}$. These patients were required to have biopsy-proven vasculitis and had to be followed by a physician who was part of the Glomerular Disease Collaborative Network ${ }^{14}$. Pagnoux and colleagues found that $54 \%$ of French patients experienced a relapse within a median followup period of 44 months ${ }^{15}$. Median time to relapse among patients with a relapse was 30 months ${ }^{15}$. Lastly, a German-based study by Holle and colleagues evaluated patients over 3 time periods: 1966-1993, 1994-1998, and 1999-2002 $2^{16}$. The first cohort was followed for an average of 6.6 years and the second for an average of 7.3 years ${ }^{16}$. During the followup, $63.9 \%$ of the first cohort and $51.2 \%$ of the second cohort experienced a relapse ${ }^{16}$. The final cohort was followed for an average of 3.9 years, during which $35.3 \%$ of patients had a relapse ${ }^{16}$.

Preventing relapses is important. From a clinical perspective, relapses indicate disease progression, and major relapses require a repetition of induction therapy ${ }^{9}$. The goal of maintenance therapy is to maintain remission and prevent disease relapse, although the optimal duration of maintenance therapy is not known ${ }^{9}$. From a patient-centered perspective, controlling disease is important because GPA and MPA can affect patients both physically and emotionally. Several analyses have found correlations between AAV and decreased quality of life, including reported depression, anxiety, and decreased general and physical health ${ }^{19,20}$. Patients requiring dialysis may experience even greater decreases in quality of life ${ }^{21}$. Lastly, relapses can be very costly as major relapses can lead to permanent organ damage and can require expensive treatments/procedures such as bronchoscopy, biopsies, dialysis, plasma exchange, and hospitalizations. In this analysis, patients with relapse had GPA-related costs nearly 4 times higher than patients who did not experience a

Personal non-commercial use only. The Journal of Rheumatology Copyright @ 2016 . All rights reserved. 


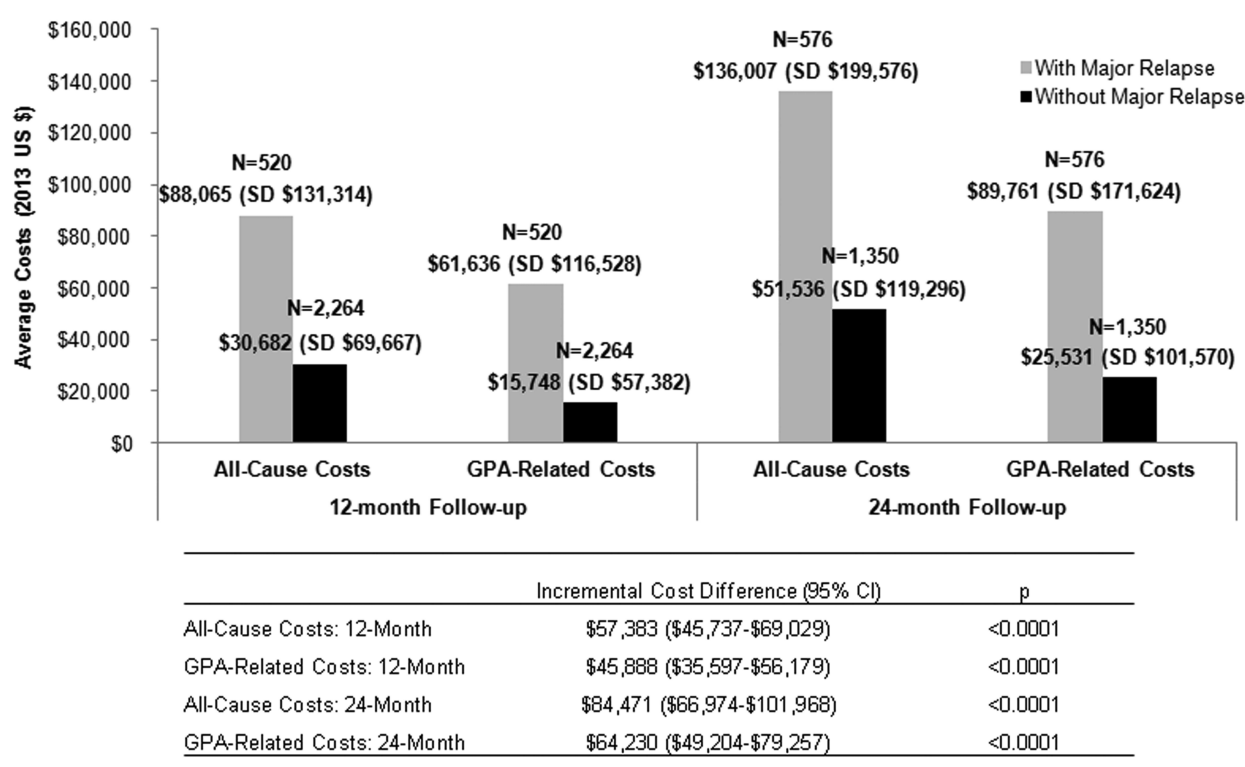

Figure 3. All-cause and GPA-related healthcare costs of patients with GPA in 12-month and 24-month followup periods by major relapse status. GPA: granulomatosis with polyangiitis.

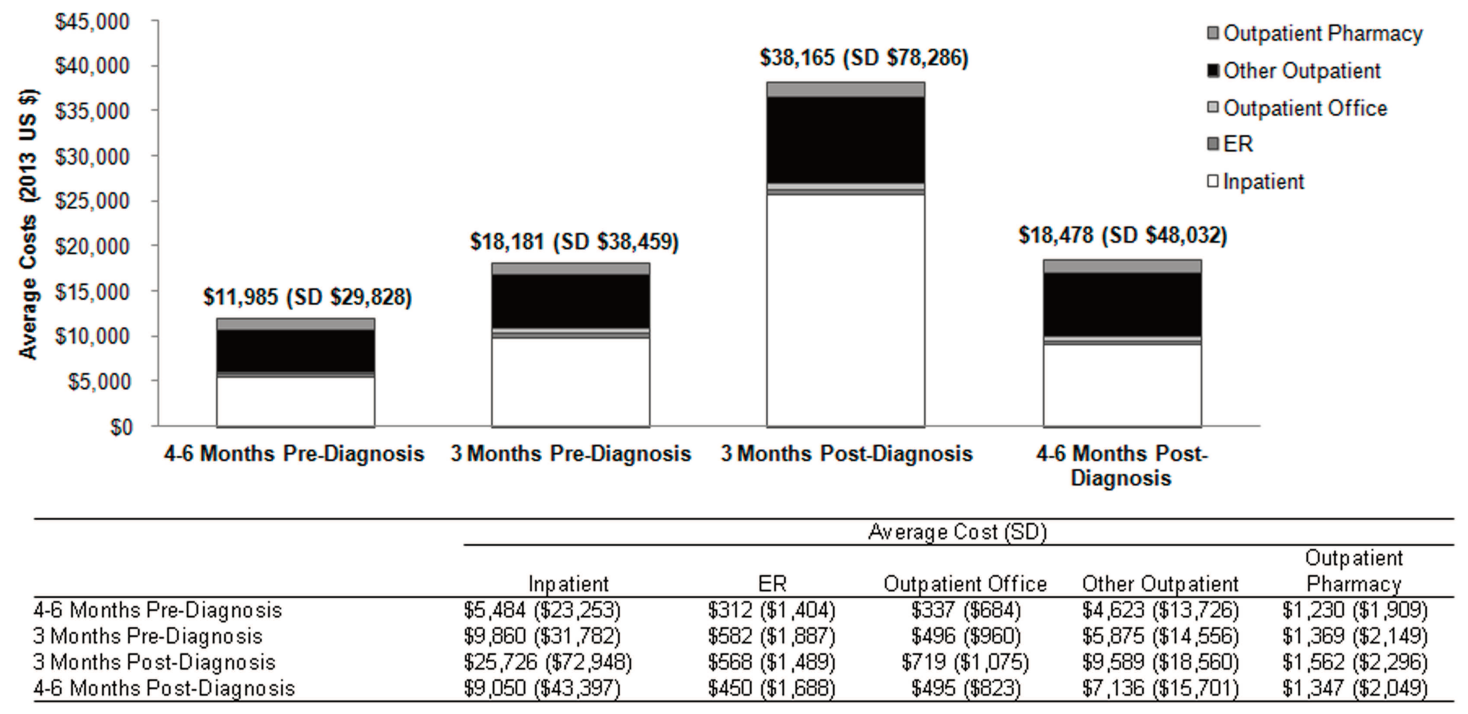

Figure 4. All-cause healthcare costs before and after MPA diagnosis. MPA: microscopic polyangiitis; ER: emergency room.

relapse. Therefore, preventing relapse is also important from an economic perspective.

Though GPA and MPA are rare conditions, they result in a high burden to the healthcare system, including hospitalizations and dialysis in the outpatient setting. Among patients with MPA, costs were particularly high immediately following diagnosis, although an increase was noted prior to diagnosis as well. To our knowledge, this is the first analysis to thoroughly describe healthcare resource use and costs for patients with GPA and MPA in the United States. Two older studies focused on inpatient admissions. In a study by Reinhold-Keller, et al, 60 German patients with GPA were interviewed about their healthcare use ${ }^{22}$. In the 12 months before the survey, 33 patients were hospitalized and the majority reported seeing a physician more than once every 3 months ${ }^{22}$. In an analysis using New York State hospitalization data and extrapolating to the United States, Cotch, et al estimated that GPA disease resulted in 12,332 hospitalizations and $\$ 148.3$ million in hospital charges ${ }^{23,24}$.

Accurate, timely diagnosis and appropriate treatment for

$$
\text { Personal non-commercial use only. The Journal of Rheumatology Copyright (C) 2016. All rights reserved. }
$$


GPA and MPA are important for managing the diseases. This analysis found that $18 \%$ of patients with incident GPA had a diagnosis for a major relapse-related condition in the 6 to 12 months prior to first GPA diagnosis. This indicates that the process to diagnosis among these patients may be lengthy because patients are experiencing serious clinical complications prior to receiving GPA diagnosis. This proportion (18\%) increased to $32.5 \%$ within the 6 months prior to diagnosis. Among patients with MPA, $60.5 \%$ had a diagnosis for a major relapse in the 6 months prior to first MPA diagnosis. This proportion is likely artificially inflated by our selection criteria, which found patients with severe disease manifestations. During the 12-month followup period, $31.5 \%$ of patients with GPA either had no claims for GPA-related therapy or were treated with corticosteroids alone. While corticosteroid use is indicated as part of some therapy regimens for induction and maintenance, use of corticosteroids alone to treat GPA is not recommended ${ }^{8}$. Therefore, those patients seemed to be inadequately treated, which may lead to an increase in relapses and in the overall healthcare burden.

Our analysis has limitations. First, it was limited to individuals with commercial health coverage or Medicare supplemental coverage. Consequently, results of this analysis may not be generalizable to patients with other insurance or without health insurance coverage. Second, there was the potential for misclassification of GPA and MPA diagnosis, incident versus prevalent diagnosis, comorbid conditions, or disease-related conditions because patients were found and variables were gathered through administrative claims data, which are collected for billing purposes rather than research. In particular, patients with MPA may be misclassified because there is no ICD-9-CM diagnosis code specific to MPA. The algorithm developed here identified patients with severe disease by requiring diagnoses of serious MPA-related manifestations. Alveolar hemorrhage could not be included in this algorithm because no ICD-9-CM diagnosis code for alveolar hemorrhage in adults exists. Therefore, we relied on the diagnosis codes for hemoptysis but this likely did not identify all patients with alveolar hemorrhage. Additionally, the number of GPA-related visits to specific provider types may have been underestimated if the physician was part of a multispecialty practice or if the provider did not put a diagnosis of GPA on the claims generated by the visit.

Misclassification or miscounting may also have occurred when identifying relapses because limited clinical information was available in the claims databases. Owing to that lack of clinical information, the definition of relapse used in this analysis differs from the definition used in clinical practice or in other analyses not based on claims. Specifically, in this analysis, remission status was not determined because the minor items on the $\mathrm{BVAS}^{7}$ may not result in a diagnosis on a claim and relapse was evaluated among all patients. Although presence of relapse was not assessed until 30 days after initial diagnosis in incident patients to avoid categorizing diagnosis as a relapse, 30 days may have been too short of a time period following initial diagnosis to achieve remission. Therefore, misclassification between initial diagnosis and relapse for incident patients may have occurred. However, the 30-day period was based on the period for evaluation specified in the BVAS instructions ${ }^{7}$. Additionally, ICD-9-CM coding is not detailed enough to say that a diagnosis of a relapse-associated condition such as stroke was necessarily a GPA-related relapse. However, given that the patient sample had GPA and were also required to start induction therapy following the diagnosis for the episode to be counted as a relapse, it may be reasonably assumed that the diagnosis was part of a relapse. Healthcare cost comparisons between patients with and without relapse were unadjusted and did not account for baseline differences between the 2 cohorts. Finally, medical services and prescription use that did not result in the generation of insurance claims, as well as inpatient drug use, were not measured in this analysis.

Because this is one of the first analyses of GPA and MPA in claims data, there are many opportunities for future research in this disease area. The sensitivity and specificity of the GPA diagnosis code should be evaluated compared to a gold standard population of patients with GPA. Similarly, the algorithm to identify patients with MPA and to identify relapses should also be validated against a gold standard and modified as necessary. Regarding costs, this analysis presented unadjusted costs, but future analysis should be conducted to compare costs between patients with and without relapse, adjusting for patient characteristics. Additionally, it may be worthwhile to estimate the incremental cost of GPA and MPA compared to healthy patients. Lastly, while this analysis focused on direct costs for patients with GPA and MPA, the indirect cost burdens of these conditions, such as lost wages, should be estimated because nearly half of the GPA population (40\%) was between 18 and 54 years of age.

In a real-world setting, patients with GPA who experience major relapses impose significant clinical and economic effects on the US healthcare system. Adoption of treatments that are more efficacious in reducing the risk of relapse may contribute to the decrease in clinical burden and total healthcare costs, improving quality of care for this population. Among patients with MPA, a new algorithm based on clinical inputs allowed for those patients to be found in a claims database. MPA diagnosis was associated with an increase in all-cause healthcare resource use and costs.

\section{ONLINE SUPPLEMENT}

Supplementary data for this article are available online at jrheum.org.

\section{REFERENCES}

1. Langford CA. Granulomatosis with polyangiitis (Wegener's). [Internet. Accessed September 8, 2015.] Available from:

Personal non-commercial use only. The Journal of Rheumatology Copyright $\subset$ 2016. All rights reserved. 
www.rheumatology.org/Practice/Clinical/Patients/Diseases_And_C onditions/Granulomatosis_with_Polyangiitis_\%28Wegener_s\%29/

2. Kallenberg CG. Key advances in the clinical approach to ANCA-associated vasculitis. Nat Rev Rheumatol 2014;10:484-93.

3. Jennette JC, Falk RJ, Bacon PA, Basu N, Cid MC, Ferrario F, et al. 2012 revised International Chapel Hill Consensus Conference Nomenclature of Vasculitides. Arthritis Rheum 2013;65:1-11.

4. Flossman O, Berden A, de Groot K, Hagen C, Harper L, Heijl C, et al. Long-term patient survival in ANCA-associated vasculitis. Ann Rheum Dis 2011;70:488-94.

5. Mahr AD. Epidemiological features of Wegener's granulomatosis and microscopic polyangiitis: two diseases or one 'anti-neutrophil cytoplasm antibodies-associated vasculitis' entity? APMIS 2009;117:14-47.

6. Zeft AS, Weiss N. Case control study of ANCA associated vasculitis in western Montana. Arthritis Rheum 2005;52:S648

7. Stone JH, Hoffman GS, Merkel PA, Min Y, Uhlfelder ML, Hellmann DB, et al. A disease-specific activity index for Wegener's granulomatosis. Arthritis Rheum 2001;44:912-20.

8. Ntatsaki E, Carruthers D, Chakravarty K, D'Cruz D, Harper L, Jayne D, et al. BSR and BHPR guideline for the management of adults with ANCA-associated vasculitis. Rheumatology 2014;53:2306-9.

9. Tarzi RM, Pusey CD. Current and future prospects in the management of granulomatosis with polyangiitis (Wegener's granulomatosis). Ther Clin Risk Manag 2014;2014:279-93.

10. Bosch X, Guilabert A, Espinosa G, Mirapeix E. Treatment of antineutrophil cytoplasmic antibody-associated vasculitis. JAMA 2007;298:655-69.

11. Stone JH, Merkel PA, Spiera R, Seo P, Langford CA, Hoffman GS, et al. Rituximab versus cyclophosphamide for ANCA-associated vasculitis. N Engl J Med 2010;2010:221-32.

12. Rituximab package insert. South San Francisco, CA: Genentech Inc.; 2014

13. Koldingsnes W, Nossent JC. Baseline features and initial treatment as predictors of remission and relapse in Wegener's granulomatosis. J Rheumatol 2003;30:80-8.

14. Hogan SL, Falk RJ, Chin H, Cai J, Jennette CE, Jennette JC, et al. Predictors of relapse and treatment resistance in antineutrophil cytoplasmic antibody-associated small-vessell vasculitis. Ann Intern Med 2005;143:621-31.
15. Pagnoux C, Hogan SL, Chin H, Jennette CE, Falk RJ, Guillevin L, et al. Predictors of treatment resistance and relapse in antineutrophil cytoplasmic antibody-associated small-vessel vasculitis: comparison of two independent cohorts. Arthritis Rheum 2008;58:2908-18.

16. Holle JU, Gross WL, Latza U, Nolle B, Ambrosch P, Heller M, et al. Improved outcome in 445 patients with Wegener's granulomatosis in a German vasculitis center over four decades. Arthritis Rheum 2011;63:257-66.

17. US Bureau of Labor. Consumer Price Index detailed report, data for January 2014, annual average tables, 2013. [Internet. Accessed September 8, 2015.] Available from: www.bls.gov/cpi/cpid1401.pdf

18. Deyo RA, Cherkin DC, Ciol MA. Adapting a clinical comorbidity index for use with ICD-9-CM administrative databases. J Clin Epidemiol 1992;45:613-9.

19. Koutantji M, Harrold E, Lane SE, Pearce S, Watts RA, Scott DG. Investigation of quality of life, mood, pain, disability, and disease status in primary systemic vasculitis. Arthritis Rheum 2003 49:826-37.

20. Carpenter DM, Thorpe CT, Lewis M, Devellis RF, Hogan SL. Health-related quality of life for patients with vasculitis and their spouses. Arthritis Rheum 2009;61:259-65.

21. Mittal SK, Ahern L, Flaster E, Maesaka JK, Fishbane S. Self-assessed physical and mental function of haemodialysis patients. Nephrol Dial Transplant 2001;16:1387-94.

22. Reinhold-Keller E, Herlyn K, Wagner-Bastmever R, Gutfleisch J, Peter HH, Raspe HH, et al. Effect of Wegener's granulomatosis on work disability, need for medical care, and quality of life in patients younger than 40 years at diagnosis. Arthritis Rheum 2002;47:320-5.

23. Cotch M, Hoffman GS, Yerg DE, Kaufman GI, Targonski P, Kaslow RA. The epidemiology of Wegener's granulomatosis. Estimates of the five-year period prevalence, annual mortality, and geographic disease distribution from population-based data sources. Arthritis Rheum 1996;39:87-92.

24. Cotch MF, Hoffman GS. The prevalence, epidemiology and cost of hospitalizations for vasculitis in New York State 1986 to 1990 [abstract]. Arthritis Rheum 1995;38:S225. 